\title{
LOS EFECTOS DEL DIVORCIO EN EL PROGENITOR CUSTODIO Y NO CUSTODIO, SEGÚN LOS JÓVENES
}

\author{
Mạa Melania Hernández García \\ Universidad Europea de Canarias \\ melania.hernandez@universidadeuropea.es \\ Beatriz Triana Pérez \\ Universidad de La Laguna
}

\section{RESUMEN}

Los agentes sociales elaboran creencias para generar expectativas sobre la realidad social en la que viven, y guiar su propia conducta. El objetivo de este estudio fue analizar la creencia explícita que tiene un grupo de jóvenes (de 19 a 26 años) sobre los efectos del divorcio en los progenitores, tras la ruptura, y si dicha creencia se ve influida por las variables que se exploran. Para ello se pasó una entrevista abierta a una muestra de 160 sujetos, distribuidos según su Experiencia personal de ruptura familiar (con experiencia vs. sin experiencia) y su Sexo (masculino vs. femenino). En general, los resultados muestran una visión más negativa que positiva sobre los efectos de la ruptura familiar en el progenitor no custodio, y lo contrario respecto a los efectos sobre el progenitor custodio. Además, la Experiencia personal de ruptura familiar es la variable que muestra mayor influencia sobre la creencia explícita acerca del divorcio y sus efectos.

Palabras clave: efectos del divorcio; progenitor custodio; progenitor no custodio; variables moduladoras; jóvenes

\section{ABSTRACT \\ Effects of divorce on the custodial and the non-custodial parent, according to young people. Social agents develop beliefs to generate expectations about the social reality in which they live, and to guide their own behavior. The aim of this study was to analyze the explicit belief held by a group of young people (from 19 to 26 years old) about the effects of divorce on the parents after the separation, and if this is influenced by the variables submitted to study. For this, an open interview was given to a sample of 160 subjects, distributed accor- ding to their personal experience of family breakdown (experienced vs. inexperienced) and their sex (male vs. female). In general, the results show a more negative than positive view of the effects of the family break in the non-custodial parent. On the contrary, a somewhat more positive than negative view of the effects on the custodial}




\section{LOS EFECTOS DEL DIVORCIO EN EL PROGENITOR CUSTODIO Y NO CUSTODIO, SEGÚN LOS JÓVENES}

parent. In addition, the Personal Experience of family breakdown is the variable that show the greatest influence on the explicit belief about divorce and its effects.

Keywords: effects of divorce; custodial parent; non-custodial parent; modulating variables; young people

\section{ANTECEDENTES}

El incremento en el número de rupturas familiares ha impulsado el estudio de los efectos que generan sobre la dinámica familiar y en la adaptación de quienes viven dicha experiencia. Así, se han observado muchos y variados efectos. En cualquier caso, quienes viven dicha experiencia, deben realizar un importante esfuerzo para poder adaptarse a la nueva realidad y circunstancias. Algunos cambios vienen asociados al tipo de custodia establecida respecto a los hijos. En este sentido, es importante señalar que últimamente se observan valores más igualitarios entre hombres y mujeres, lo que redunda en un impulso cada vez más notorio en pro de la coparentalidad. No obstante, a pesar de ello, las estadísticas muestran todavía hoy una clara preponderancia de custodias en solitario ejercidas por las madres. Ello implica que muchos hijos e hijas crecen en contextos de ruptura familiar con experiencias dispares para las madres y los padres, construyendo creencias concretas sobre dichas realidades.

La literatura científica ha explorado dicho tema, observándose determinados rasgos asociados a la custodia en solitario, como es el sufrir mayor estrés por la atención de los hijos (Pruett, Williams, Insabella y Little, 2003) 0 el menor tiempo disponible para sociabilizar y/o rehacer su vida afectiva (Cantón, Cortés y Justicia, 2007; Castells, 2009), entre otros. También se observan efectos positivos como el mantener un contacto próximo e intenso con sus hijos, fortaleciendo así las relaciones entre ellos (Muñoz, 2014; Riggio, 2004). Por su parte, los progenitores no custodios ganan en libertad y oportunidades para el ocio o para rehacer su vida afectiva (Hetherington y Kelly, 2005), pero tienen que enfrentar mayor número de cambios en su vida cotidiana (Guttmann, 1993), y ven reducido el contacto con sus hijos, pudiendo ello provocar un enfriamiento en sus relaciones (Hilton y Kopera-Frye, 2006). También existen efectos comunes para ambos, como sufrir problemas emocionales (Wallerstein y Lewis, 2005; Yárnoz-Yaben, 2013), cambios económicos (Morgado, Jiménez-Lagares y González, 2007; Ozawa y Yoon, 2003), o nuevas dificultades entre ellos (Fischer, De Graaf y Kalmijn, 2005; Sbarra y Emery, 2005), etc.

Muchos de los efectos negativos encontrados se reducen en los casos de custodia compartida, o en custodias en solitario donde los progenitores sostienen relaciones respetuosas entre ellos, exentas de hostilidad, esforzándose por desarrollar una coparentalidad positiva que facilite la adaptación de sus hijos. Ello sería el principal objetivo cuando se produce una ruptura familiar.

Pues bien, teniendo en cuenta que el divorcio es un evento frecuente que afecta cada año a un número considerable de familias españolas, no es descabellado pensar que muchos de los jóvenes de hoy en día puedan vivenciar, en el futuro, dicha experiencia. Por ello, es importante conocer sus creencias acerca del divorcio y sus efectos en los progenitores, atendiendo a los tipos de custodia posibles, en solitario o compartida. Un mayor conocimiento sobre los efectos diferenciales sobre el custodio y el no custodio, y el reconocimiento de los beneficios de la custodia compartida o de una custodia en solitario que sea flexible, puede lograr que en el futuro se beneficien todos los miembros de un núcleo familiar que rompe, al permitirles alcanzar mejores dinámicas tras la ruptura, facilitando además una mayor presencialidad de ambos progenitores en la vida de sus hijos e hijas.

Así, en el presente trabajo se exploran las creencias explícitas de un grupo de jóvenes sobre los efectos positivos y los efectos negativos que viven los progenitores custodios y no custodios tras la ruptura familiar, para conocer el grado de conocimiento que tienen al respecto, y si dicha información se acerca a la realidad. Además, se explora si dichas creencias difieren en función de la experiencia personal de ruptura familiar y el sexo de los participantes. Esta información es relevante para tenerla en cuenta en los casos de mediación ante situaciones de ruptura, ya que a veces, la falta de un conocimiento real, o de perspectivismo, puede conllevar dificultades para alcanzar acuerdos. Dicho trabajo fue en parte subvencionado con el proyecto del Ministerio de Economía y Competitividad, referencia EDU2012-38588 y, además, se engloba dentro de las acciones desarrolladas dentro de Centro de Estudios Universitarios de la Mediación y la Convivencia (CUMECO). 


\section{MÉTODO}

\section{Muestra}

En el estudio participaron 160 jóvenes, con edades comprendidas entre los 19 y los 26 años, cuya media de edad era de 22,40 años (Desviación Típica 2,32). La muestra se distribuye equitativamente atendiendo a variables como el grado de experiencia en ruptura familiar en sus familias de origen (50\% divorciadas/separadas; $50 \%$ sin ruptura) y el sexo (50\% hombres, $50 \%$ mujeres).

\section{Instrumento}

Se elaboró un cuestionario semiestructurado para recoger la información. En el primer bloque se exploran numerosos datos sociodemográficos acerca del participante, su familia y la experiencia que tienen en ruptura familiar. En el segundo, se presentan diversas cuestiones abiertas acerca del divorcio y sus efectos, positivos y negativos, sobre los distintos miembros de la familia. Para este estudio se seleccionó la información referida a los progenitores, según su condición de custodio y no custodio, con el fin de apresar la creencia explícita de los participantes sobre el tema.

\section{Procedimiento}

Se contactó con alumnado de Psicología para facilitar la búsqueda de participantes, utilizando, para ello, el procedimiento denominado bola de nieve. Tras contactar con los participantes e informarles sobre la tarea a realizar, solicitando su colaboración voluntaria, se les proporcionó el material para que rellenaran, de forma autónoma, el cuestionario. Tras la recogida de la información, se seleccionó para el estudio una muestra cuyos perfiles permitieran equiparar los grupos de contraste en relación con las variables independientes a explorar, pero también, intentando controlar el posible influjo de otras variables.

\section{Diseño de investigación y análisis de los datos}

Se trata de un estudio cuasi-experimental, de corte transversal con muestreo no probabilístico subjetivo de conveniencia, basado en un diseño factorial inter-grupos con variables cruzadas 2 (con experiencia en ruptura familiar, sin experiencia en ruptura familiar) $\times 2$ (hombre, mujer).

El tratamiento de los datos parte de la extracción inicial de las categorías, para cada cuestión a analizar, y la posterior codificación de la información, mediante un sistema de dos jueces, obteniéndose porcentajes de acuerdo altamente satisfactorios en los casos (Kappa superiores a .90). Para el tratamiento estadístico se seleccionaron las dimensiones de respuesta, para cada contenido, cuya frecuencia fuera superior, al menos en uno de los niveles de la variable independiente examinada en cada caso, a lo que se podría dar por azar, atendiendo a la prueba de aproximación de la distribución binomial a la curva normal, con un error típico alfa del 5\%.

Las variables dependientes son los porcentajes de citación de las diferentes dimensiones de respuesta seleccionadas, para cada cuestión. Al ser variables tipo Dummy (toman valores 0 si no se nombran, 01 cuando sí se nombran), implican que dichos porcentajes son equivalentes a las medias, permitiendo su tratamiento estadístico a través del modelo lineal general univariante (ANOVAs). Los datos se analizan con el paquete estadístico SPSS versión 25, presentando datos descriptivos, ANOVAs y pruebas "t" de Student para analizar las interacciones entre variables, y el estadístico de Cohen $\left(\eta^{2}\right)$ para conocer el tamaño de los efectos encontrados. También se utiliza la "t" de Student en la comparación de los resultados observados respecto al custodio y al no custodio.

\section{RESULTADOS}

En este apartado se describen los principales resultados obtenidos en el análisis de las creencias de los jóvenes sobre los cambios positivos y negativos que sufren los progenitores custodio y no custodio, tras una ruptura. Además, se exploran los posibles efectos de las variables independientes exploradas, sobre dichas creencias. 


\section{LOS EFECTOS DEL DIVORCIO EN EL PROGENITOR CUSTODIO Y NO CUSTODIO, SEGÚN LOS JÓVENES}

\section{Efectos positivos en el progenitor custodio y no custodio}

La Figura 1 recoge el porcentaje de participantes que citan cada categoría de respuesta ante la pregunta ¿Cuáles son los cambios positivos en el progenitor custodio y no custodio, tras la separación de la pareja? Se señalan también los contrastes que han salido significativos al comparar los porcentajes referidos al progenitor custodio y al no custodio.

Los resultados muestran respuestas diseminadas en diferentes categorías, tanto en relación con el custodio como con el no custodio. La única respuesta que obtiene un mayor consenso, en ambos casos, es la mejora emocional tras la separación.

En relación con el custodio se citan también, aunque en menor grado, las respuestas Intensifica la atención dedicada a sus hijos, Incrementa el contacto con sus hijos, Tiene más libertad para hacer las cosas que le gustan, o Se siente feliz al quedarse con la custodia. En relación con el progenitor no custodio destacan también las respuestas Tiene más libertad para hacer las cosas que le gustan, Inicia una nueva relación afectiva y Sufre menor estrés y responsabilidades. En porcentajes inferiores también citan Intensifica la atención dedicada a sus hijos, Tiene más tiempo libre y No sé.

Figura 1. Porcentaje de citación de cada efecto positivo atribuido por los participantes al progenitor custodio y al no custodio, tras una ruptura.

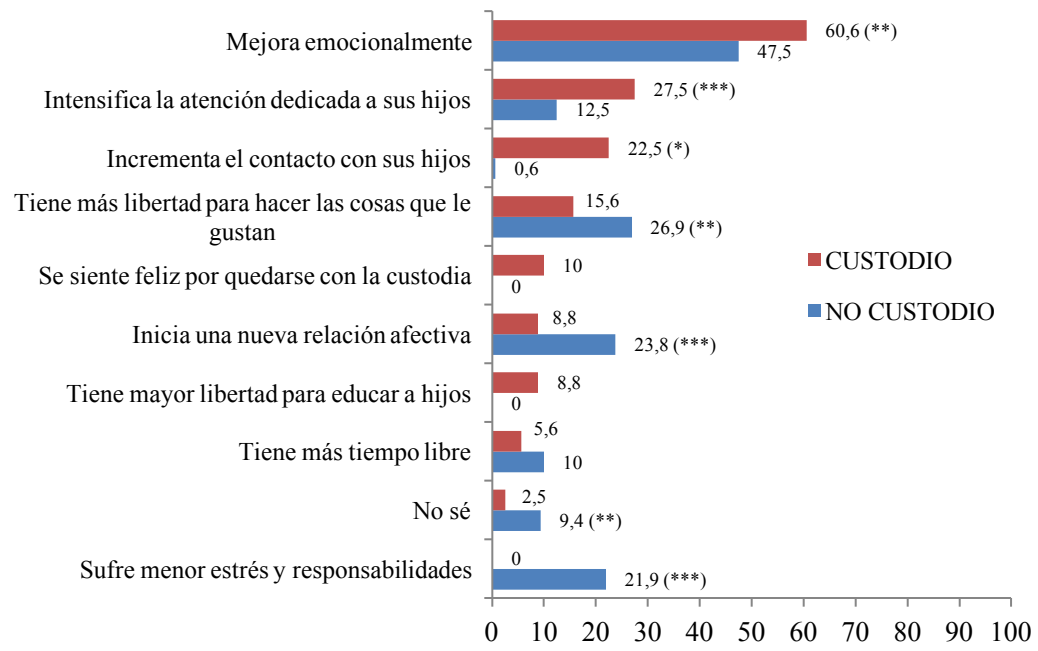

Nota: $(*) \mathrm{p} \leq .05(* *) \mathrm{p} \leq .01(* * *) \mathrm{p} \leq .001$

Comparando ahora las medias de citación de cada dimensión de respuesta referida al custodio y al no custodio, se observan diferencias significativas en la categorías siguientes: Mejora emocionalmente $(\mathrm{t}(159)=2,84$, $\mathrm{p}=.005)$, Intensifica la atención dedicada a sus hijos $(\mathrm{t}(159)=3,67, \mathrm{p}=.000)$, Incrementa el contacto con sus hijos $(\mathrm{t}(159)=2,51, \mathrm{p}=.013)$, Tiene más libertad para hacer las cosas que le gustan $(\mathrm{t}(159)=2,84, \mathrm{p}=.005)$, Inicia una nueva relación afectiva $(\mathrm{t}(159)=4,66, \mathrm{p}=.000)$ y Sufre menor estrés y responsabilidades $(\mathrm{t}(159)=6,67, \mathrm{p}=.000)$. No se observaron diferencias significativas en las dimensiones restantes.

\section{Influjo de las variables independientes en los efectos positivos}

Se explora ahora si las variables independientes influyen sobre el grado de citación de los distintos efectos positivos atribuidos al progenitor custodio y al no custodio. La Tabla 1 recoge los efectos principales obtenidas 
en Ios ANOVAs realizados, y el grado del efecto asociado ( ${ }^{2}$ parcial). No se han obtenido efectos significativos en las interacciones entre las variables independientes, para ninguna de las variables dependientes exploradas.

La Experiencia de ruptura familiar, en relación con el progenitor custodio, muestra dos diferencias significativas para las categorías Intensifica la atención dedicada a sus hijos $\left(\mathrm{F}_{(1,159)}=4,59, \mathrm{p}=.034\right)$ y Se siente feliz por

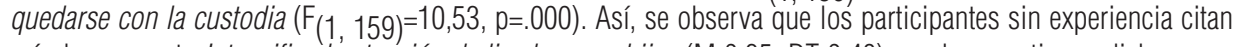
más la respuesta Intensifica la atención dedicada a sus hijos (M 0,35; DT 0,48) que los que tienen dicha experiencia (M 0,20; DT 0,40), con un grado del efecto pequeño; y también la categoría Se siente feliz por quedarse con la custodia (M 0,18; DT 0,38) frente a los que sí tienen experiencia (M 0,03; DT 0,16), en este caso el grado del efecto es mediano.

En relación al progenitor no custodio, se encontró una diferencia significativa para la respuesta Tiene más tiempo libre $\left(F_{(1,159)}=7,17, p=.008\right)$, siendo los participantes sin experiencia quienes más la citan (M 0,16; DT 0,37 ) frente a los que tienen dicha experiencia (M 0,04; DT 0,19), con un tamaño del efecto pequeño.

Tabla 1.

ANOVAs significativos, y tamaño de los efectos, respecto a los efectos positivos en el progenitor custodio y en el no custodio, según las variables independientes exploradas.

\begin{tabular}{|c|c|c|c|c|c|c|}
\hline \multirow{2}{*}{ Progenitor custodio } & \multicolumn{3}{|c|}{ Experiencia de ruptura } & \multicolumn{3}{|c|}{ Sexo } \\
\hline & $(F)$ & $(p)$ & $\left(\eta^{2}\right)$ & $(F)$ & (p) & $\left(\eta^{2}\right)$ \\
\hline $\begin{array}{l}\text { Intensifica la atención dedicada a } \\
\text { sus hijos }\end{array}$ & 4,59 & $.34(*)$ & $.03(\bullet)$ & & & \\
\hline $\begin{array}{l}\text { Se siente feliz por quedarse con la } \\
\text { custodia }\end{array}$ & 10.53 & $.000(* * *)$ & $.07(\bullet \bullet)$ & & & \\
\hline $\begin{array}{l}\text { Tiene más libertad para hacer lo } \\
\text { que le gusta }\end{array}$ & & & & 5.88 & $.16(*)$ & $.04(\bullet)$ \\
\hline $\begin{array}{l}\text { Tiene mayor libertad para educar a } \\
\text { hijos }\end{array}$ & & & & 5.11 & $.25(*)$ & $.03(\bullet)$ \\
\hline \multirow{2}{*}{ Progenitor no custodio } & \multicolumn{3}{|c|}{ Experiencia de ruptura } & \multicolumn{3}{|c|}{ Sexo } \\
\hline & $(F)$ & $(p)$ & $\left(\eta^{2}\right)$ & $(F)$ & $(p)$ & $\left(\eta^{2}\right)$ \\
\hline Mejora emocionalmente & & & & 5.01 & $.27(*)$ & $.03(\bullet)$ \\
\hline Tiene más tiempo libre & 7.17 & $.008(* *)$ & $.04(\bullet)$ & & & \\
\hline
\end{tabular}

Nota. $(*) \mathrm{p}<.05(* *) \mathrm{p}<.01(* * *) \mathrm{p}<.001 ; \eta^{2}(\bullet)$ Efecto pequeño $(\bullet \bullet)$ Efecto mediano

La variable independiente Sexo del participante también muestra dos efectos principales para el progenitor custodio, a modo de tendencias, sobre los cambios Tiene más libertad para hacer las cosas que le gustan $\left(\mathrm{F}_{(1}\right.$, $159)=5,88, p=.016)$ y Tiene mayor libertad para educar a sus hijos $\left(F_{(1,159)}=5,11, p=.025\right)$. Los contrastes muestran que las chicas citan más ambos cambios (M 0,23; DT 0,42 y M'0,14; DT 0,35) frente a los chicos (M 0,09; DT 0,28 y M 0,04; DT 0,19, respectivamente).

En cuanto al progenitor no custodio, dicha variable tan sólo muestra un efecto principal sobre el cambio

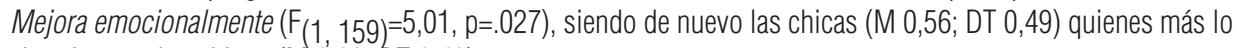
citan frente a los chicos (M 0,39; DT 0,49).

\section{Efectos negativos en el progenitor custodio y no custodio}

En la Figura 2, se recoge el porcentaje de participantes que citan cada categoría de respuesta en relación ahora con los cambios negativos para el custodio y el no custodio, tras la ruptura. Se señalan también los contrastes que han salido significativos al comparar los porcentajes referidos al progenitor custodio y al no custodio. 


\section{LOS EFECTOS DEL DIVORCIO EN EL PROGENITOR CUSTODIO Y NO CUSTODIO, SEGÚN LOS JÓVENES}

Figura 2. Porcentaje de citación de cada efecto negativo atribuido por los participantes al progenitor custodio y al no custodio, tras una ruptura.

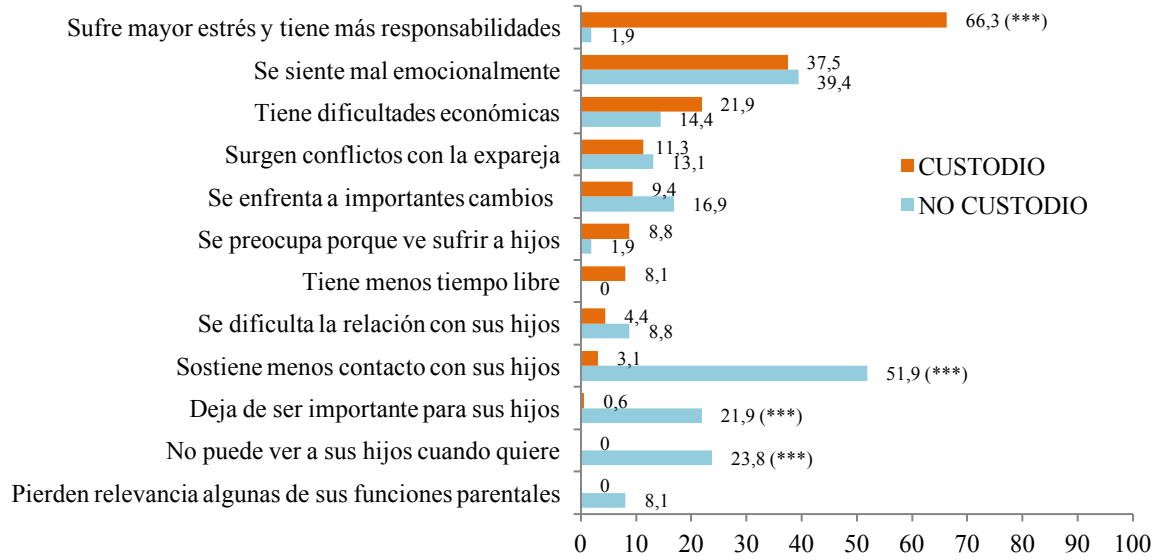

Nota: $(* * *) \mathrm{p} \leq .001$

Al igual que lo observado con los cambios positivos, las respuestas se encuentran diseminadas en diferentes categorías. Respecto al progenitor custodio, destaca especialmente el efecto Sufre mayor estrés y tiene más responsabilidades. En menor grado sobresale también Se siente mal emocionalmente y Tiene dificultades económicas. Con porcentajes menores también citan Surgen conflictos con la expareja, Se enfrenta a importantes cambios o Tiene menos tiempo libre. Respecto al progenitor no custodio, nombran, principalmente, el efecto Sostiene menos contacto con sus hijos. Además de éste, destaca Se siente mal emocionalmente, No puede ver a sus hijos cuando quiere y Deja de ser importante para sus hijos. En menor grado también nombran Se enfrenta a importantes cambios, Tiene dificultades económicas, Surgen conflictos con la expareja, Se dificulta la relación con los hijos y Pierden relevancia en algunas de sus funciones parentales.

Los contrastes de medias mediante la prueba "t" de Student muestran diferencias significativas tan solo para Ios cambios Sufre mayor estrés y tiene más responsabilidades(t $(159)=16,51, p=.000)$, Sostiene menos contacto con sus hijos (t(159)=11,73, $\mathrm{p}=.000)$, Deja de ser importante para sus hijos $(\mathrm{t}(159)=6,32, \mathrm{p}=.000)$ y No puede ver a sus hijos cuando quiere $(t(159)=5,92, p=.000)$.

\section{Influjo de las variables independientes en los efectos negativos}

La Tabla 2 recoge Ios ANOVAs que han salido significativos, y el grado del efecto ( 2 parcial), cuando se explora el posible efecto de las variables independientes estudiadas, sobre las variables dependientes. 
Tabla 2.

ANOVAs significativos, y tamaño de los efectos, respecto a los efectos negativos en el progenitor custodio y en el no custodio, según las variables independientes exploradas.

\begin{tabular}{llcc}
\hline \multirow{2}{*}{ Progenitor custodio } & \multicolumn{3}{c}{ Experiencia de ruptura } \\
\cline { 2 - 4 } & $(F)$ & $(p)$ & $\left(\eta^{2}\right)$ \\
\hline $\begin{array}{l}\text { Sufre mayor estrés y tiene más } \\
\text { responsabilidades }\end{array}$ & 25.08 & $.000(* * *)$ & $.15(\bullet \bullet)$ \\
Se siente mal emocionalmente & 7.04 & $.009(* *)$ & $.05(\bullet)$ \\
\hline \multirow{2}{*}{ Progenitor no custodio } & \multicolumn{3}{c}{ Experiencia de ruptura } \\
\cline { 2 - 4 } & 4.49 & $(p)$ & $\left(\eta^{2}\right)$ \\
\hline Se siente mal emocionalmente & 9.71 & $.036(*)$ & $.03(\bullet)$ \\
Surgen conflictos con la expareja & 20.34 & $.000(* * *)$ & $.07(\bullet \bullet)$ \\
Dejtiene menos contacto con sus hijos & $.12(\bullet \bullet)$ \\
Pierden relevancia en sus funciones & 10.68 & $.004(* *)$ & $.05(\bullet)$ \\
parentales & & & $.07(\bullet *)$ \\
\hline
\end{tabular}

Nota. $(*) \mathrm{p}<.05(* *) \mathrm{p}<.01(* * *) \mathrm{p}<.001 ; \eta^{2}(\bullet)$ Efecto pequeño $(\bullet \bullet)$ Efecto mediano $(\bullet \bullet)$ Efecto grande

Los resultados muestran que tan sólo la variable Experiencia personal de ruptura familiar ejerce efectos sobre las variables dependientes. Con referencia al progenitor custodio, Ios ANOVAs muestran diferencias significativas para las categorías Sufre mayor estrés y tiene más responsabilidades $(F(1,159)=25,08, p=.000)$, y Se siente mal

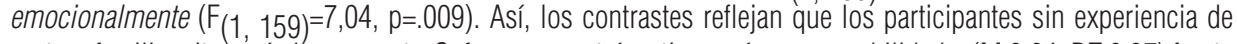
ruptura familiar citan más la respuesta Sufre mayor estrés y tiene más responsabilidades ( $\mathrm{M} 0,84$; DT 0,37) frente a quienes sí tienen experiencia (M 0,49; DT 0,50); mientras que los participantes con experiencia citan más Se siente mal emocionalmente (M 0,48; DT 0,50) frente a los que no tienen experiencia (M 0,28; DT 0,45).

En relación al progenitor no custodio, se encontraron diferencias significativas para las respuestas Se siente mal emocionalmente $\left(F_{(1,159)}=4,49, p=.036\right)$, Surgen conflictos con la expareja $(F(1,159)=9,71, p=.002)$, Sostiene menos contacto con sus hijos $\left(F_{(1,159)}=20,34, p=.000\right)$, Deja de ser importante para sus hijos $\left(F_{(1}\right.$, $159)=8,57, p=.004)$ y Pierden relevancia algunas de sus funciones parentales $\left(F_{(1,159)}=10,68, p=.000\right)$. Así, se observa que los participantes sin experiencia citan más que los que tienen experiencia las categorías Se siente mal emocionalmente (M 0,48; DT 0,50 versus M 0,31; DT 0,47), Surgen conflictos con la expareja (M 0,21; DT 0,41 versus $\mathrm{M}$ 0,05; DT 0,22), Sostiene menos contacto con sus hijos (M 0,69; DT 0,47 versus M 0,35; DT 0,48), Deja de ser importante para sus hijos (M 0,31; DT 0,47 versus M 0,13; DT 0,33) y Pierden relevancia algunas de sus funciones parentales (M 0,15; DT 0,36 versus M 0,01; DT 0,11).

\section{DISCUSIÓN}

En España, numerosas familias con hijos se divorcian cada año, muchas de manera amistosa, y alrededor de un tercio, por la vía contenciosa. Los servicios de Mediación cobran especial relevancia en estas ocasiones, facilitando que las parejas Ileguen a acuerdos respecto a decisiones que van a condicionar de forma significativa sus vidas, tras la ruptura (p.e., el tipo de custodia, el régimen de comunicación con sus hijos...) (Parkinson, 2005). Un conocimiento objetivo sobre cómo puede afectar la ruptura para los miembros de la familia, según el tipo de custodia, puede ayudar a los implicados a moderar sus posturas y tratar de alcanzar acuerdos, especialmente para beneficiar a los hijos. 


\section{LOS EFECTOS DEL DIVORCIO EN EL PROGENITOR CUSTODIO Y NO CUSTODIO, SEGÚN LOS JÓVENES}

El trabajo que se presenta ha pretendido conocer las creencias de un grupo de jóvenes sobre los efectos de la ruptura en los progenitores porque, probablemente, un porcentaje importante de ellos vivirán dicha experiencia en el futuro. Así, el estudio se centró en el análisis de los efectos positivos y negativos de la ruptura sobre el progenitor custodio y el no custodio, cuando se opta por la custodia en solitario.

Los resultados parecen mostrar una actitud más positiva que negativa hacia la ruptura, ya que un porcentaje importante de jóvenes señala, como efecto relevante, la mejora emocional de quienes se separan, frente a los que afirman lo contrario. No obstante, se observa que las creencias de los participantes se soportan en una variedad de efectos positivos y negativos escasamente consensuados, si atendemos a los porcentajes con los que son citados. Así, los jóvenes nombran, además de la mejora emocional, y también como aspectos positivos atribuidos significativamente más al progenitor custodio, el hecho de que éste intensifica la atención y el contacto con sus hijos, y que se siente feliz por asumir la custodia. También nombran tener más libertad para hacer cosas que les gusten, pero este efecto se atribuye significativamente más al no custodio. A este último le atribuyen también, y en mayor grado, la posibilidad de rehacer su vida afectiva, el sufrir menor estrés y responsabilidades, y también la respuesta no sé. Estos efectos ya han sido encontrados en algunos estudios que analizan la dinámica de las familias divorciadas, como se señaló en la introducción (Hetherington y Kelly, 2005; Muñoz, 2014; Riggio, 2004).

Los efectos negativos ofrecen una pauta similar en cuanto a la diversidad de categorías de respuesta encontradas, pero en este caso, algunas de ellas muestran mayor robustez según el progenitor evaluado. Así, más de tres quintas partes de los participantes atribuyen mayor estrés y responsabilidades al custodio, por ser el principal encargado de la atención y cuidado de los hijos; mientras que algo más de la mitad de los participantes atribuyen un menor contacto con los hijos al no custodio, la menor importancia de su papel ante ellos, y la dificultad para verlos cuando les apetece. Todos los efectos se citan significativamente más para los personajes comentados. Ello indica una elaboración de realidades diferentes para cada uno de ellos. Además, las dificultades económicas, el surgimiento de nuevos conflictos entre la expareja, el hecho de enfrentarse a nuevos cambios, etc., son también cambios reconocidos por un porcentaje pequeño de jóvenes respecto a ambas figuras. También numerosos estudios encuentran muchos de los efectos nombrados (Ozawa y Yoon, 2003; Sbarra y Emery, 2005; Yárnoz-Yaben, 2013).

El mayor número de respuestas no sé, y el deterioro percibido en varias respuestas respecto a las relaciones del no custodio con sus hijos, ofrecen una versión más negativa de las relaciones paternofiliales, por ser los padres quienes asumen principalmente el rol del no custodio. Ello refleja un mensaje poco prometedor en relación con el cambio social hacia la igualdad, ya que realza significativamente el papel de los custodios, normalmente las madres, en detrimento del de los padres.

Por su parte, el análisis del influjo de las variables independientes pone de manifiesto que es la experiencia de haber vivido o no la ruptura en el entorno personal, lo que afecta principalmente a las creencias encontradas. Al respecto, los jóvenes sin experiencia citan más que el custodio se siente más feliz al recibir dicha responsabilidad, pero sufre más estrés por dicha razón; o que el no custodio sostiene menos contacto con sus hijos y pierde relevancia su papel ante ellos, o que suele enfrentar nuevos conflictos con sus exparejas. A ello se suman otros efectos que salen significativos, pero a modo de tendencia. Lo mismo ocurre en relación con la variable sexo, donde las chicas destacan por encima de los chicos algunos efectos, pero tan solo como tendencias.

La falta de homogeneidad y robustez en las respuestas encontradas podría significar que los jóvenes reflejan la gran heterogeneidad de experiencias que se observan en la realidad. Pero, más específicamente, podría indicar la escasa elaboración que hacen sobre el divorcio y sus efectos, y la asunción de un estereotipo más marcado en quienes no tienen experiencia de ruptura familiar. Estos resultados muestran, por tanto, la necesidad de una mayor formación en los jóvenes y en la sociedad, sobre los efectos del divorcio y las variables que favorecen transiciones que permitan alcanzar mejores niveles de adaptación para quienes viven dichas experiencias. 


\section{REFERENCIAS BIBLIOGRÁFICAS}

Cantón, J., Cortés, M., y Justicia, M. (2007). Conflictos entre los padres, divorcio y desarrollo de los hijos. Madrid: Pirámide.

Castells, P. (2009). Los padres no se divorcian de sus hijos. Madrid: Aguilar.

Fischer, T., De Graaf, P., y Kalmijn, M. (2005). Friendly and antagonistic contact between former spouses after divorce. Journal of Family Issues, 26(8), 1131-1163.

Guttmann, J. (1993). Divorce in psychosocial perspective: theory and research. London: Lea.

Hetherington, E., y Kelly, J. (2005). En lo bueno y en lo malo: Ia experiencia del divorcio. Cómo influye realmente la separación en la vida de padres e hijos. Barcelona: Paidós.

Hilton, J., y Kopera-Frye, K. (2006). Loss and depression in cohabiting and noncohabiting custodial single parent. The Family Journal: Counseling and Therapy for Couples and Families, 14(1), 28-40.

Morgado, B., Jiménez-Lagares, I., y González, M. (2007). Familias tras separación o divorcio. Experiencia y ajuste psicológico. Letras de Deusto, 115(37), 93-107.

Muñoz, D. (2014). Por mis hijos lo que sea... o casi. Identidades de madres y padres que han vivido un divorcio. Papeles del CEIC, 1, 63-92.

Ozawa, M., y Yoon, H. (2003). Economic impact of marital disruption on children. Children and Youth Services Review, 25(8), 611-632.

Parkinson, L. (2005). Mediación Familiar. Barcelona: Editorial Gedisa.

Pruett, M., Williams, T., Insabella, G., y Little, T. (2003). Family and legal indicators of child adjustment to divorce among families with young children. Journal of Family Psychology, 17(2), 169-180.

Riggio, H. (2004). Parental marital conflict and divorce, parent-child relationships, social support, and relationship anxiety in young adulthood. Personal Relationships, 11, 99-114.

Sbarra, D., y Emery, R. (2005). Coparenting conflict, nonacceptance, and depression among divorced adults: results from a 12-year follow-up study of child custody mediation using multiple imputation. American Journal of Orthopychiatry, 75(1), 63-75.

Wallerstein, J., y Lewis, J. (2005). The reality of divorce. Psychoanalytic Psychology, 22(3), 452-454.

Yárnoz-Yaben, S. (2013). What if it was all a mistake? Negative thoughts and adjustment to divorce. Estudios de Psicología, 34(2). 
